\title{
A pain in the arms
}

\author{
Loredana Catalano $\cdot$ Antonio Perrone • \\ Carmela Cecere $\cdot$ Carlo Sabbà
}

Received: 16 October 2013/Accepted: 15 November 2013/Published online: 19 December 2013

(C) SIMI 2013

\section{Case presentation}

Dr Catalano: In October 2011, a 73-year-old shoemaker was admitted to the chest pain unit of a provincial hospital for atypical chest pain that arose a few hours earlier. He had a history of ischemic heart disease, and had an acute myocardial infarction at the age of 65 , and had a subsequent coronary artery bypass graft. He also had peripheral edema and with bilateral upper limb functional weakness and progressively more severe myalgia. These other symptoms had arisen about 2 weeks prior, and with growing intensity. Blood tests excluded an acute coronary syndrome (troponin negative), but showed the presence of an elevated serum creatinine phosphokinase (CPK). During hospitalization in the chest pain unit, the patient also underwent an echocardiographic examination, which showed the presence of moderate heart disease. A few days later, the patient was transferred to our unit because of worsening bilateral edema and myalgia of the upper limbs. There was no family history of muscular disorders, but only hypertension and diabetes mellitus (DM). He reported that at the age of 53 years, a monoclonal gammopathy had been diagnosed, and 1 year later arterial hypertension.

He had been under treatment for several years with betablockers, acetyl salicylic acid, antihypertensives, nitrates, proton pump inhibitors as well as simvastatin $(20 \mathrm{mg} / \mathrm{day})$, but had never shown clinical or biochemical findings of muscle toxicity. On visual examination, the patient was afebrile but the arms, especially the forearms, were red and

L. Catalano $(\square) \cdot$ A. Perrone $\cdot$ C. Cecere · C. Sabbà Dipartimento di Mdecina Interna "C. Frugoni",

Università degli Studi di Bari, Policlinico Universitario,

Piazza G. Cesare 1, 70124 Bari, Italy

e-mail: loredanacatal@hotmail.it swollen with diffuse bilateral hard edema and marked weakness. The blood pressure was $120 / 80 \mathrm{mmHg}$, heart rate 80 beats/min., and no other physical signs were present.

Dr Cecere: We carried out blood tests that were normal except for a CPK 2504 U/L (nv: 21-232), lactic dehydrogenase (LDH) $305 \mathrm{U} / \mathrm{L}$ (vn: 100-190), creatine kinase MB (CK-MB) $5.1 \mathrm{U} / \mathrm{L}$ (nv up to 3.6), myoglobin $707 \mathrm{ng} / \mathrm{ml}$ (vn: 16-96), erythrocyte sedimentation rate $33 \mathrm{~mm} / \mathrm{h}$ (vn: 1-10), fibrinogen $745 \mathrm{mg} / \mathrm{dl}(170-410)$, serum glutamic oxaloacetic transaminase $210 \mathrm{U} / \mathrm{L}$ (vn: 15-37), serum glutamic pyruvic transaminase $84 \mathrm{U} / \mathrm{L}$ (nv 12-78), hemoglobin $11.3 \mathrm{~g} / \mathrm{dl}$ (nv 12-15). Urinalysis showed moderate hemoglobin concentration with no sign of red blood cells on microscopic examination. This was hypothesized to be due to the presence of myoglobinuria.

\section{Differential diagnosis}

Dr Perrone: At the beginning, we hypothesized the presence of a mediastinal syndrome in view of the bilateral edema of the arms, and decided to perform a computerized tomography (CT) scan of the chest that was negative, as were also heteroplastic markers. Subsequently we paid close attention to the high levels of CPK, LDH, CK-MB, Myoglobin and the presence of myoglobinuria, and hypothesized that this was due to the presence of rhabdomyolysis. We investigated several causes of rhabdomyolysis (Table 1): intense muscular activity, damage, drugs, infections, immunopathological disease, systemic amyloidosis. The patient declared that he had not suffered any muscle strain due to excessive physical effort, did not drink excessive alcohol, and had not suffered recent trauma. Serum viral tests were negative, autoantibodies (anti-nuclear antibody, extractable nuclear antigens, 
Table 1 Causes of rhabdomyolysis

\begin{tabular}{|c|c|c|}
\hline Acquired acute causes & Acquired chronic causes & Congenital causes \\
\hline $\begin{array}{l}\text { Intense muscular activity } \\
\text { Exercise } \\
\text { Status epilepticus } \\
\text { Tetanus } \\
\text { Delirium tremens }\end{array}$ & $\begin{array}{l}\text { Immunopathological disease } \\
\text { Dermatomyositis } \\
\text { Polymyositis }\end{array}$ & $\begin{array}{l}\text { Abnormalities of lipid metabolism } \\
\text { Carnitine deficiency } \\
\text { CPT deficiency }\end{array}$ \\
\hline $\begin{array}{l}\text { Direct muscle damage } \\
\text { Trauma } \\
\text { Burns }\end{array}$ & $\begin{array}{l}\text { Metabolic disorders } \\
\text { Diabetes mellitus } \\
\text { Primary aldosteronism } \\
\text { Renal tubular acidosis } \\
\text { Myxedema } \\
\text { Parenteral nutrition }\end{array}$ & $\begin{array}{l}\text { Abnormalities of carbohydrates metabolism } \\
\text { Myophosphorylase deficiency } \\
\alpha \text {-glucosidase deficiency } \\
\text { Amilo } 6 \text { glucosidase deficiency } \\
\text { Fosforisomerasi } \\
\text { Phosphofructokinase deficiency }\end{array}$ \\
\hline $\begin{array}{l}\text { Muscle ischemia } \\
\text { Compression } \\
\text { Vascular occlusion } \\
\text { Sickle cell anemia } \\
\text { Embolism }\end{array}$ & $\begin{array}{l}\text { Others } \\
\text { Systemic amyloidosis } \\
\text { Idiopathic }\end{array}$ & Muscular dystrophies \\
\hline $\begin{array}{l}\text { Infections } \\
\text { (viral, bacterial, other) }\end{array}$ & & \\
\hline \multicolumn{3}{|l|}{ Drugs } \\
\hline $\begin{array}{l}\text { Ethanol } \\
\text { Methadone, heroin }\end{array}$ & & \\
\hline Cyclosporine, steroids & & \\
\hline Overdose of salicylates & & \\
\hline Diuretics, amphotericin & & \\
\hline Liquorice, statins & & \\
\hline Biological toxins & & \\
\hline Lithium, antipsychotics & & \\
\hline Electrolyte disturbances & & \\
\hline Hypokalemia & & \\
\hline Hypophosphatemia & & \\
\hline Hypo/hypernatremia & & \\
\hline
\end{tabular}

anti-neutrophil cytoplasmic antibodies, anti-liver kidney microsomal antibodies, anti smooth muscle antibodies, anti mitochondria antibodies) were all negative, while anti hepatitis $\mathrm{C}$ virus antibodies (anti-HCV) was positive (HCV-RNA $=562,407$ copies), and cryoglobulins were absent. Serum immunofixation showed the presence of $\mathrm{IgG}$ monoclonal component $\mathrm{k}$, negative Bence Jones protein in urine. thyroid-stimulating hormone (TSH) was $10.2 \mathrm{mIU} / \mathrm{L}$ (vn: 0.3-3.6), free thyroxine (FT4): $1.10 \mathrm{ng} / \mathrm{dl}$ (vn:

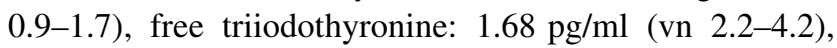
anti thyroglobulin antibodies and anti thyroid peroxidase antibodies normal. During hospitalization, a total body CT scan was performed, and was negative, while EchoDoppler examination of the upper limbs showed: the absence of venous thrombosis bilaterally, soft tissue edema and slowing of venous outflow.
A bone marrow biopsy showed a picture compatible with MGUS but Congo red staining was negative. Radiographic examination of the skeleton revealed no osteolytic lesions. The statin therapy was immediately suspended.

\section{Diagnosis}

Dr Catalano, Dr Cecere: He was diagnosed with a "spontaneous compartment syndrome (CS) of the upper limbs with rhabdomyolysis, and likely iatrogenic hypothyroidism associated with paraproteinaemia $\operatorname{IgG} \mathrm{k}$ ". Given the finding of hypothyroidism, treatment with thyroid replacement therapy was prescribed (L-thyroxine at a dose of $25 \mathrm{mg} /$ day, which was subsequently increased to 
$50 \mathrm{mg} /$ day), as well as with anti-edema drugs (methylprednisolone at a dose of $0.5 \mathrm{mg} / \mathrm{kg} /$ day, later reduced), hydrating saline as well as alkaline diuretics, which produced a marked clinical and biohumoral improvement in a few days. Muscle enzymes returned to normal after about a week. After normalization of the thyroid function, we decided to reintroduce the statin therapy 2 months later. Subsequent blood tests showed no signs of rhabdomyolysis.

\section{Case discussion}

Prof. Sabbà, Dr Catalano: Fractures are the most common cause (up to about $70 \%$ ) of CS of the limbs. In the remaining $20 \%$ of CS, the disease is a direct consequence of tissue injury (crush syndrome, exercise, arterial injury, etc.), or in some rare cases it may occur spontaneously like a complication of low molecular weight heparin therapy [1]. The disease develops when the pressure within the compartment (ICP: intra compartmental pressure) exceeds the normal capillary filling pressure $(12-32 \mathrm{mmHg}$ ), while the normal pressure in the tissue compartments ranges between 0 and $8 \mathrm{mmHg}$. The syndrome is normally correlated with an impaired capillary blood flow at pressures of about $20 \mathrm{~mm} \mathrm{Hg}$, and the patient feels pain already at pressures of between 20 and $30 \mathrm{~mm} \mathrm{Hg}$, while ischemia develops at higher pressures. If left untreated, the ischemia can have dramatic consequences for the patient such as necrosis of the limbs, which may be life-threatening and necessitate amputation. This syndrome can affect any area of the body with little or no expansion capacity of the tissues. The diagnosis is mainly based on clinical findings. The characteristic seven "P"s of the CS [1] (pain, pressure, poikilothermia, paralysis, paresthesia, pulselessness, pallor) may be useful, while laboratory and instrumental investigations (e.g., Doppler ultrasound) can help but are not decisive for diagnostic purposes. The gold standard in the diagnosis is surely the measurement of the ICP although emergency departments are rarely equipped with the tools for this procedure. The clinical presentation of CS is mainly in an acute-onset form, but a subacute and sometimes chronic onset have also been described. In acute forms, an early diagnosis is essential, before the muscle damage becomes irreversible. In these cases, emergency fasciotomy cannot be deferred, and is the only feasible treatment to prevent a fatal outcome.

There are many causes of both acute and chronic CS (Table 2). As shown by data in the literature, systemic rhabdomyolysis rarely leads to a spontaneous CS exclusively affecting both forearms, as observed in our patient. The onset of a spontaneous CS is often associated with DM, hypothyroidism, viral myositis, leukemic infiltration, nephrotic syndrome, a ruptured aneurysm or cyst or prolonged use of anticoagulants. The patient was in treatment with statins (simvastatin), at low doses, but in any case immediate preventive suspension of the treatment was judged advisable. Therapy with CoA inhibitors (statins) is used as primary and secondary prevention of coronary artery disease, as well as being strongly recommended in the guidelines as the first choice treatment of hypercholesterolemia. Although generally well tolerated and with few side effects, such as headache and gastrointestinal disorders, therapy with statins may be limited by side effects on the skeletal muscle such as myalgia, and rarely rhabdomyolysis. The risk of statins inducing a myopathy or rhabdomyolysis seems to increase with age, dosage (>40 mg/day), DM, liver dysfunction, chronic renal failure, a low BMI, hypothyroidism, alcohol consumption and the use of other drugs (e.g., macrolides), which may alter the pharmacokinetics in competition with cytochrome $\mathrm{P} 450$ (CYP) 3A4 [2]. In fact, the percentage of muscle pathologies seems to be correlated with high doses of statins (but our patient was only taking low doses) and their plasma

Table 2 Causes of compartment syndrome

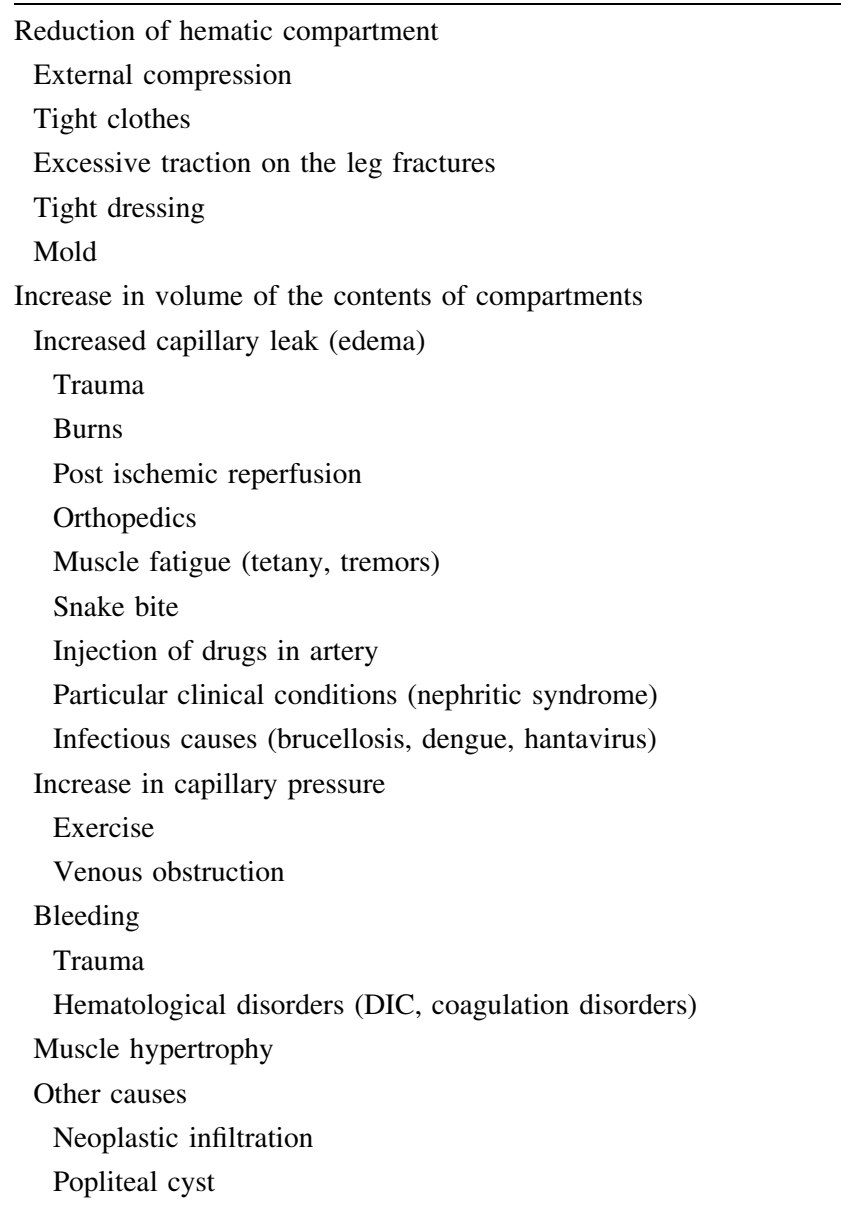

Modified by Nau and Matsen (31-32) 
concentrations [3]. The underlying pathophysiological mechanism is unknown and is probably multifactorial [4]. Several theories have proposed that statin myotoxicity may be due to the depletion of intracellular cholesterol or to interference with the oxidative phosphorylation pathway. The precise mechanisms have yet to be fully defined. In several studies, a $0.02 \%$ risk of rhabdomyolysis for doses of $20 \mathrm{mg} /$ day of statins (simvastatin) has been reported (in particular, these studies refer to simvastatin), $0.07 \%$ for doses of $40 \mathrm{mg} / \mathrm{day}$ and $0.3 \%$ for doses of $80 \mathrm{mg} / \mathrm{day}$. In our patient, we believe that a decisive role was played by the association with hypothyroidism, even if only subclinical, given the values of TSH and FT4. Hypothyroidism is a common cause of mild-to-moderate hyper-CPKemia. Only in severe cases may severe rhabdomyolysis be observed. Several cases of rhabdomyolysis-induced hypothyroidism have been reported [5], but mostly precipitated by exercise. Only rarely have cases of rhabdomyolysis induced by statins been reported in the literature, but these patients were not in treatment for an associated but probably undiagnosed hypothyroidism [6]. In our patient, treatment with thyroid replacement therapy resulted in complete remission of symptoms. We conclude that hypothyroidism probably triggered the systemic rhabdomyolysis and consequent CS confined to the upper limbs, since the introduction of thyroid replacement therapy resulted in a rapid improvement of symptoms (about a week). In this case, CS was due to the additive effect of different conditions. It is very important to carry out screening of thyroid function before starting statin therapy to reduce the risk of serious complications. The subsequent reintroduction in our patient of statin treatment after discharge at the previous dosages did not induce any symptoms of muscle strain, as confirmed by subsequent laboratory tests. Hypothyroidism is the most common thyroid disease that often goes undiagnosed. From the epidemiological point of view, it has the same frequency as DM type II (approximately $5 \%$ of the population, and yet media interest in the two diseases is very different) [7]. We justified the rare location of CS in the upper limbs by the fact that the family of repetitive motion disorders (RMD) also belongs to the category of spontaneous forms of CSs, namely those diseases (trauma disorders, biomechanical muscle overload diseases, occupational syndromes, hand-arm vibration syndrome, overuse syndrome, etc.) resulting from excessive physical strain that require an adequate period of rest and recovery; all together, these account for $60 \%$ of occupational diseases. Our patient had been a shoemaker for many years. The anatomical region under the greatest stress in this type of manual activity is the anterior compartment of the forearm.

The basic pathophysiological mechanisms underlying the development of RMD are:
1. Muscle tension, that beyond certain limits can cause circulation alterations and thus lead to metabolic and degenerative diseases,

2. Repetitive motion,

3. A non-ergonomic posture causing excessive muscle strain with possible spasm and compression of the internal structures [8].

Physiologically, during exertion, the muscle volume increases by $20-30 \%$ of its initial volume. This is due to both the mechanical effect of muscle contraction and to the passage of fluid from the vessels into the interstitium. When the muscles are working in optimal perfusion and aerobic conditions, the intra-muscular pressure (IMP) returns to normal between contractions because there is a rapid reabsorption of interstitial fluids. However, when the muscles are subjected to excessive or repeated stress, in the absence of adequate energy reserves or training for such physical effort, this may result in a deficient reabsorption of interstitial fluids and hence an increased muscle volume [9]. Normally, this process promotes a physiological increase in muscle mass, as occurs in the course of training. But if this process does not occur in a linear, progressive manner, beyond a certain critical threshold of adaptation, it can lead to a conflict between the rigidity of the muscle-tendon aponeurosis on one hand and the circulatory flow and muscle volume on the other. This is more common in subjects with predisposing factors of an anatomical (if the aponeurosis is too thick or too stiff, or circulation or muscle alterations), metabolic (decompensated DM, electrolyte imbalances), or mechanical nature (as in our shoemaker). Under these conditions, a minimal increase in muscle volume may be enough to cause a massive increase in the IMP [10] leading to obstruction of the microvessels and a delayed/blocked muscle perfusion.

\section{The importance of screening}

Prof Sabbà: In conclusion, the case we report demonstrates the importance of serial screening both of CPK levels and thyroid function before and during treatment with statins, even if administered at low dosages, to prevent serious musculo-systemic complications. Excessive, repeated strain of the forearms established the local conditions for the onset of a CS, an unusual condition that is rarely observed in clinical practice.

Conflict of interest None.

\section{References}

1. Adelman Leon C, McGillicuddy Daniel C (2012) Enoxaparin associated atraumatic compartment syndrome of the calf. Intern Emerg Med 7(Suppl 2):S81-S83 
2. Barahona MJ, Mauri A, Sucuzza N, Paredes R, Wanger AM (2002) Hypothyroidism as a cause of Rhabdomyolysis. Endocr J 49:621-623

3. Faiza AQ (2009) Severe rhabdomyolysis and acute renal failure secondary to use of simvastatin in undiagnosed hypothyroidism. Saudi J Kidney Dis Transpl 20:127-129

4. Ronaldson KJ, O'Shea JM, Boyd IW (2006) Risk factors for rhabdomyolysis with simvastatin and atorvastatin. Drug Saf 29(12): 1991

5. Riggs JE (1990) Acute exertional rhabdomyolysis in hypothyroidism: The result of reversible defect in glycogenolysis. Mil Med 155:171-172
6. Thompson PD, Clarkson P, Karas RH (2003) Statin-associated myopathy. JAMA 289:1681-1690

7. Yeter Ekrem, Keles Telat, Durmaz Tahir, Bozkurt Engin (2007) Rhabdomyolysis due to the additive effect of statin therapy and hypothyroidism: a case report. J Med Case Rep 1:130

8. Andersoon R (1988) Chronic compartment syndrome of the forearm extensor. Lakartiduinger 85:218

9. Artuson G, Kjiellmer I (1964) Capillary permeability in skeletal muscle during rest and activity. Acta Phisiol Scand 62:41

10. Sodeberg TA (1996) Bilateral chronic compartment syndrome of the forearm and the hand. J Bone Jt Surg 78(B):780-782 